\title{
Detecting phonons and persistent currents in toroidal Bose-Einstein condensates by means of pattern formation
}

\author{
M. Modugno, ${ }^{1,2}$ C. Tozzo, ${ }^{3}$ and F. Dalfovo ${ }^{1,4}$ \\ ${ }^{1}$ CNR-INFM BEC Center, I-38050 Povo, Trento, Italy \\ ${ }^{2}$ LENS and Dipartimento di Matematica Applicata, Università di Firenze, \\ via Nello Carrara 1, I-50019 Sesto Fiorentino, Italy \\ ${ }^{3}$ Scuola Normale Superiore, I-56126 Pisa, Italy \\ ${ }^{4}$ Dipartimento di Fisica, Università di Trento, I-38050 Povo, Italy
}

(Dated: June 13, 2021)

\begin{abstract}
We theoretically investigate the dynamic properties of a Bose-Einstein condensate in a toroidal trap. A periodic modulation of the transverse confinement is shown to produce a density pattern due to parametric amplification of phonon pairs. By imaging the density distribution after free expansion one obtains i) a precise determination of the Bogoliubov spectrum and ii) a sensitive detection of quantized circulation in the torus. The parametric amplification is also sensitive to thermal and quantum fluctuations.
\end{abstract}

PACS numbers: 03.75.Lm, 03.75.Kk, 67.40.Vs

Bose-Einstein condensates have recently been obtained with ultracold gases in a ring-shaped geometry [1]. Other groups are proposing different techniques to get toroidal condensates [2]. The aim is to create a system in which fundamental properties, like quantized circulation and persistent currents, matter-wave interference, propagation of sound waves and solitons, can be observed in a clean and controllable way. An important issue concerns the feasibility of high-sensitivity rotation sensors.

In this work we show that key properties of condensates in toroidal traps can be measured by means of parametric amplification. This corresponds to an exponential growth of some excited modes of the system induced by a periodic modulation of an external parameter [3]. We consider the modulation of the transverse confinement, which is shown to drive a spatially periodic pattern in both density and velocity distributions as a consequence of the amplification of pairs of counter-rotating Bogoliubov phonons. If the trap is switched off, this pattern produces a peculiar flower-like density distribution of the freely expanding gas. The number of "petals" and their shape provide a sensitive measure of the excitation spectrum and the superfluid rotation of the condensate.

We perform numerical simulations by integrating the Gross-Pitaevskii equation [4] for $N$ bosonic atoms of mass $M$, confined in an external potential $V_{\text {ext }}$ :

$$
i \hbar \partial_{t} \psi=\left[-\frac{\hbar^{2}}{2 M} \nabla^{2}+V_{\mathrm{ext}}+g|\psi|^{2}\right] \psi
$$

The mean-field coupling constant is given by $g=$ $4 \pi \hbar^{2} a / M$, where the s-wave scattering length $a$ is assumed to be positive. The order parameter of the condensate, $\psi(\mathbf{r}, t)$, is normalized to $\int d \mathbf{r}|\psi|^{2}=N$ and may by written as $\psi=n^{1 / 2} \exp (i S)$, where $n$ is the condensate density and the phase $S$ is related to the superfluid velocity by $\mathbf{v}=(\hbar / M) \nabla S$. We consider a condensate of $N=2 \times 10^{5}$ atoms of ${ }^{87} \mathrm{Rb}$ confined in a torus of length
$L=2 \pi R=100 \mu \mathrm{m}$ by an axially symmetric potential which has a minimum, $V_{\text {ext }}=0$, at $z=0$ and $r_{\perp}=R$. The trap is harmonic and isotropic in the $\left(z, r_{\perp}\right)$-plane around this minimum, with frequency $\omega_{\perp}=2 \pi \times 1 \mathrm{kHz}$. With this choice the transverse width of the condensate, $r_{0}$, is significantly smaller than the radius of the torus. This implies that curvature effects are almost negligible in the ground state and in the in-trap dynamics and one can replace the torus of radius $R$ with a cylinder of length $L$ with periodic boundary conditions [5]. Curvature effects are instead important during the free expansion of the condensate, and therefore the full toroidal geometry is used for simulating the dynamics after the release from the trap. The choice made for the geometry and the parameters is intended to simulate feasible experiments, but the effects we are going to show can be obtained with different types of traps and in a wide range of parameters.

The process that we want to study has three steps: i) the condensate is initially prepared in the torus; ii) the transverse harmonic potential is periodically modulated for a time $t_{\text {mod }}$; iii) both the trap and the modulation are switched off and the gas freely expands for a time $t_{\exp }$.

We first solve the stationary GP equation and the Bogoliubov equations obtained by linearizing Eq. (1) [4] for a cylindrical condensate, by using the same numerical techniques as in [6]. We obtain the stationary solution $\psi_{0}(\mathbf{r})$ and its excitation spectrum, namely the eigenfrequencies $\omega_{i}$ and quasiparticle amplitudes $u_{i}(\mathbf{r})$ and $v_{i}(\mathbf{r})$. The condensate at step (i) is assumed to be at equilibrium. Quantum and/or thermal fluctuations are included by means of the Wigner representation of bosonic fields [7]. In practice, the function $\psi$, input of step (ii), is taken of the form $\psi=\psi_{0}+\sum_{i}\left[c_{i} u_{i}+c_{i}^{*} v_{i}^{*}\right]$, where the sum extends over a wide set of Bogoliubov states including those which are expected to be relevant for the subsequent parametric amplification. The Wigner representation enters through the choice of the 


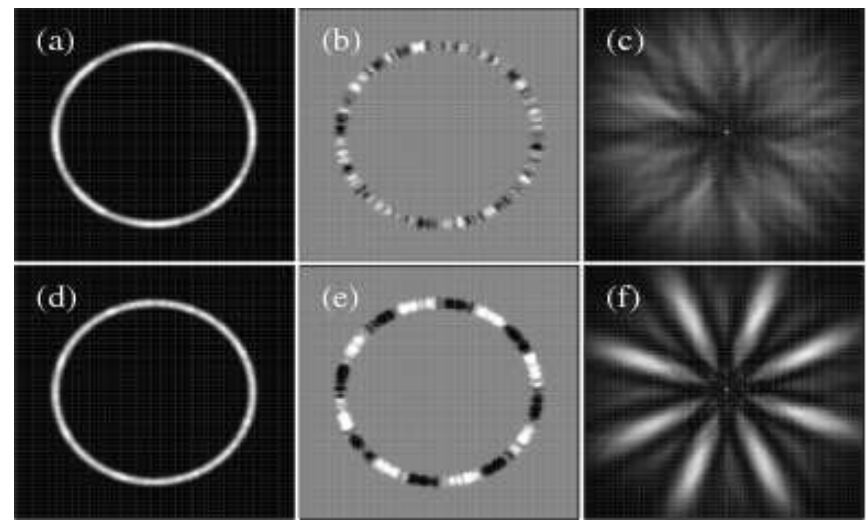

FIG. 1: a) Density of a condensate in the toroidal trap after a transverse modulation with $\Omega=0.6 \omega_{\perp}, A=0.1$, and $t_{\text {mod }}=130 \mathrm{~ms}$, corresponding to a maximum of visibility of the density pattern; the density exhibits 8 oscillations and the relative density variation is about $20 \%$. b) Tangential current for the same condensate. c) Density after $7 \mathrm{~ms}$ of free expansion. d-e-f) Same as above but for $t_{\text {mod }}=130.8 \mathrm{~ms}$, when the pattern visibility is maximum in the current distribution. Each box is $45 \mu \mathrm{m}$ per side. Densities and currents are integrated along the axis perpendicular to the torus. White (black) means high (zero) density, while in frames (b) and (e) white and black correspond to currents in opposite directions.

complex coefficients $c_{i}$, which are taken to have a randomly distributed phase, with constant probability in $[0,2 \pi)$, and modulus, with Gaussian distribution of width $1 / 2+\left[1-\exp \left(\hbar \omega_{i} / k_{B} T\right)\right]^{-1}$.

To simulate the in-trap dynamics at step (ii) we numerically integrate Eq. (11) [8]. The external modulation is included by multiplying $V_{\text {ext }}$ by the factor $f(t)=$ $[1+s(t) A \cos (\Omega t)]$, where $A$ is the amplitude of the modulation and $s(t)$ is an appropriate switching function. A collective motion which could be easily excited by this perturbation is the radial breathing mode of frequency $2 \omega_{\perp}[9]$. However, the processes that are of interest here are those involving longitudinal phonons at frequency lower than $2 \omega_{\perp}$. We also want that the transverse width and the density of the condensate oscillate in-phase with the external modulation. To this aim, the possible coupling with the breathing mode has to be suppressed. This can be obtained by choosing $s(t)$ to be sufficiently adiabatic. In the following we will use an amplitude $A=0.1$, for which we find that a switching time of about $20 \mathrm{~ms}$ is adequate in a wide range of $\Omega$. The entire modulation time, $t_{\mathrm{mod}}$, is instead chosen to be longer than $100 \mathrm{~ms}$.

The transverse modulation is found to induce a longitudinal pattern formation [10] corresponding to a standing wave along the torus. An example is shown in Fig. 1(a) where the in-trap density distribution is plotted for a modulation of frequency $\Omega=0.6 \omega_{\perp}$ and duration $t_{\text {mod }}=130 \mathrm{~ms}$. In this case the density oscillations along the torus have 8 maxima and correspond to a relative density variation of about $20 \%$. This pattern is found to occur via the parametric instability of Bogoliubov phonons, with wavevector $k$, propagating along the torus in opposite directions. The mechanism is basically the same already described in [11, 12] for elongated condensates in a modulated 1D optical lattice and it is analogous to the spontaneous pattern formation discussed in [13] in different geometries. It has also interesting similarities with the phenomenon of Faraday's instability [10, 14] for classical fluids in annular resonators [15].

The spatial periodicity of the standing wave in the torus is fixed by the wavelength of the most rapidly growing modes, determined by the Bogoliubov dispersion relation $\omega(k)$ and the boundary conditions. The parametric amplification spontaneously selects modes with frequency close to the resonance condition $\omega(k)=\Omega / 2$ and wave vector $k=2 \pi m / L$, where the integer $m$ represents the azimuthal angular momentum of the excitation and $2 m$ is the number of nodes in the density pattern. The position of the nodes changes randomly at each realization, being related to the phase of the initial fluctuations which are parametrically amplified.

At resonance the $\pm k$ components of the Fourier transform of the order parameter along the torus, $\tilde{\psi}\left(k, r_{\perp}\right)$, grow exponentially during the modulation. Since the GP equation (11) contains a nonlinear mean-field term, the growth is limited by the energy transfer to nonresonant modes due to mode-mixing processes. Thus the $\pm k$ components eventually saturates around a maximum value for long times. An example is given in the upper panel of Fig. 2, where we show the quantity $P_{k}=2 \pi \int d r_{\perp} r_{\perp}\left|\tilde{\psi}\left(k, r_{\perp}\right)\right|^{2}$ as a function of $t_{\bmod }$ for $\Omega=0.6 \omega_{\perp}$. In the lower panel we plot the maximum value achieved by $P_{k}$ in case of a modulation with $t_{\text {mod }}$ up to $200 \mathrm{~ms}$, as a function of $\Omega$. The position of each peak coincides with twice the frequency of the corresponding $m$-phonon, as calculated by solving the Bogoliubov equations. The figure suggests the possibility to use the parametric amplification for spectroscopy: Each time a density pattern is observed, the wavevector $k$ is simply obtained by counting the number of oscillations in the torus, while the frequency $\omega(k)$ is just $\Omega / 2[16]$.

The standing density wave is phase-locked with the external modulation: the amplitude of density oscillations is maximum when the width of the condensate is either maximum or minimum, and vanishes when the width is the same of the unperturbed condensate. This also causes the fast oscillation of the quantity $P_{k}(t)$ in Fig. 2. The plots in Fig. 1(a) and (d) correspond to a maximum and a minimum of $P_{k}(t)$, respectively; the density fluctuations in Fig. 1(d) are random and small. The standing density wave is accompanied by a pattern in the velocity distribution. This is easily seen by plotting the tangential current $J \equiv(i \hbar / 2 M)\left[\psi\left(\psi^{*}\right)^{\prime}-(\psi)^{\prime} \psi^{*}\right]$, as we did in Fig. 1(b) and (e): the velocity distribution exhibits small random fluctuations in (b) and a clean periodic pattern in (e). The density and velocity distributions oscillate 

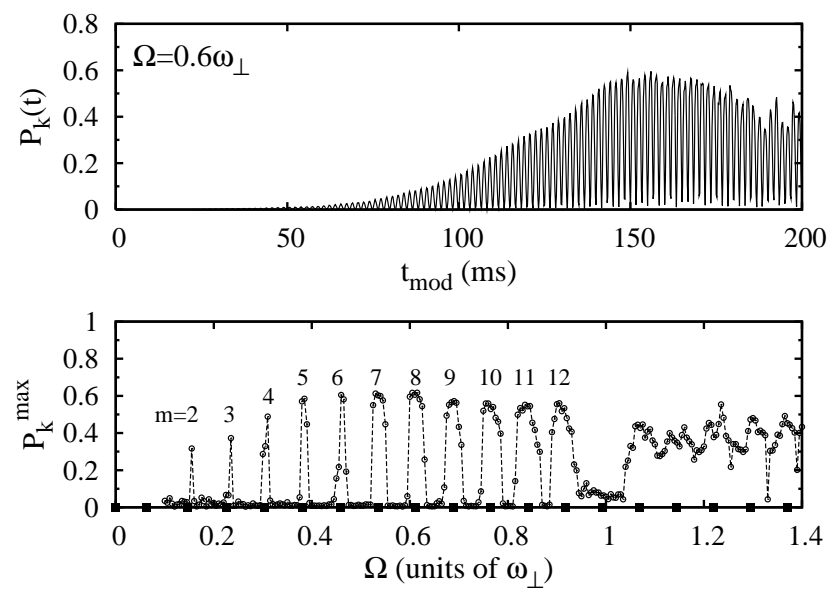

FIG. 2: Top: Amplitude of the $\pm k$ components of the Fourier transform of the order parameter along the torus, where the wavevector $k$ satisfies the resonance condition $\omega(k)=\Omega / 2$. The amplitude is plotted as a function of $t_{\text {mod }}$ for $\Omega=0.6 \omega_{\perp}$ and $A=0.1$. Bottom: maximum value of $P_{k}$ in the interval $0<t_{\mathrm{mod}}<200 \mathrm{~ms}$ as a function of $\Omega$ (in units of $\omega_{\perp}$ ). Solid squares on the horizontal axis correspond to twice the eigenfrequencies $\omega(k)$ of the Bogoliubov equations, with $k=$ $2 \pi m / L$ and $m$ integer.

exactly out-of-phase in time.

The occurrence of a pattern in the velocity field has spectacular consequences in the expansion after the release from the trap. In fact, the presence of $\pm k$-phonons gives rise to interference fringes of atoms expanding in preferred directions, similar to those observed in the expansion of an elongated condensate with Bragg excited phonons [17]. In toroidal geometry these fringes produce a flower-like structure with $m$ "petals" reflecting the periodicity of the initial velocities. An example is given in Fig. 1(c) and (f) where we show what happens to the condensates of Fig. 1(a) and (d), respectively, when the trap is switched off and the gas is imaged after $7 \mathrm{~ms}$ of free expansion. The figure shows that, by starting the expansion when the pattern has maximum amplitude in velocity field, one obtains very clean interference fringes. Since these "petals" are much more visible than the intrap density oscillations, the expansion significantly enhances the sensitivity of the spectroscopic measurement.

The dynamics in Fig. 1(c) and (f) is simulated by solving Eq. (1) without the external potential and neglecting the mean-field interaction. From the experimental viewpoint, this situation corresponds to either tuning the scattering length $a$ to zero by means of a Feshbach resonance just before the expansion or starting from a torus strongly squeezed in the $z$-direction. In the latter case, due to the fast expansion along $z$, the density decreases much faster than the typical timescale for the transverse expansion, thus suppressing mean-field effects in the radial motion. However the choice to neglect the meanfield interaction is not necessary for obtaining a distinct

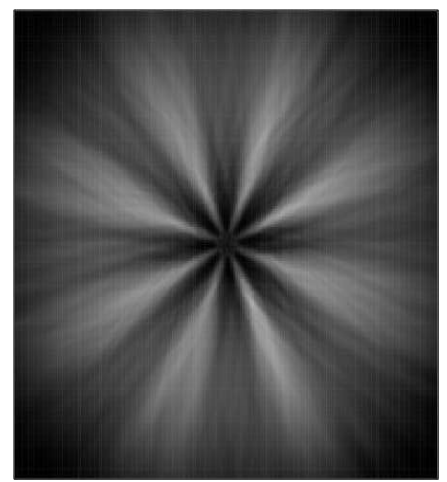

FIG. 3: Density of the expanded condensate as in Fig. 1(f). The expansion starts again from the condensate in Fig. 1](d), but here the mean-field interaction is included in the simulation of the expansion, while in Fig. 1(f) it is neglected. The expansion time is $t_{\mathrm{exp}}=1.8 \mathrm{~ms}$. The box is $45 \mu \mathrm{m}$ per side.

flower-like structure. This can be seen in Fig. 3 where we plot the density after free expansion including the meanfield interaction for a condensate prepared as in Fig.1(d); the petals are still visible also in this case. The main difference with Fig. 1(f) is the timescale of the expansion, which is faster in the presence of the repulsive mean-field interaction. A rough estimate of the ratio of the two timescales, which holds at short times, is easily obtained by comparing the expansion of a cylindrical condensate in these two cases: i) the scaling behavior of the full GP equation [4] and ii) the dispersion of a noninteracting wavepacket with the same initial shape. Using the parameters of our condensate, the interacting gas turns out to expand faster by a factor $\gamma \simeq 3.3$. In a toroidal geometry this factor is expected to be slightly larger. The ratio between the two expansion times in Fig. 1 and Fig. 3 has been taken to be $\simeq 4$ and the size of the expanding condensate and of the petals is indeed similar.

The process of pattern formation is significantly affected by the presence of quantized circulation. If the condensate is initially rotating with angular momentum $L_{z}=\kappa \hbar$ per particle, where $\kappa$ is the quantum of circulation, then the external modulation produces a pattern which rotates along the torus at the same angular velocity of the condensate as a result of the frequency shift of counter-rotating phonons [18]. More strikingly, the interference structure observed in the free expansion exhibits a significant misalignment of opposite petals, proportional to $\kappa$. This is evident in Fig. 团 where we show the same expanded condensate but for different values of $\kappa$. The axis of each petal can be approximated with a rectilinear segment tangent to a circle of radius $R_{0}$, so that $2 R_{0}$ is the distance between the axis of opposite petals. The radius $R_{0}$ is of the order of the size of a $\kappa$-vortex core in the expanding condensate. Its time evolution is well reproduced by the law $R_{0}(t)=R \kappa \alpha+\beta \kappa t / R$. The second term, with $\beta$ of order 1 , is consistent with the asymptotic 

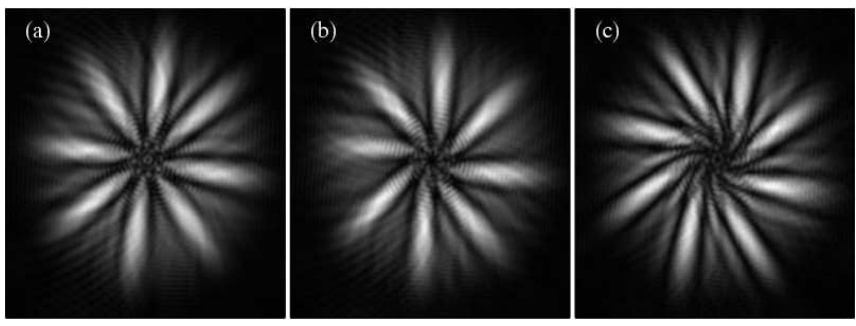

FIG. 4: Interference patterns in the expansion of a parametrically excited condensate in the presence of quantized circulation $(\kappa=1,5,10$ from $(a)$ to $(c))$. The superfluid rotation causes a misalignment of the fringes proportional to $\kappa$. In all plots $t_{\exp }=7 \mathrm{~ms}$ and $t_{\mathrm{mod}}=180.8 \mathrm{~ms}$. Each box is $55 \mu \mathrm{m}$ per side.

expression for the vortex profile that can be derived analytically in the case of free expansion as discussed in [19]. The quantity $\kappa \alpha$ in the first term is of the order of the ratio between the rotational velocity of the condensate in the torus and the velocity at which the condensate expands radially; this ratio is $\kappa \alpha \sim(\hbar \kappa / M R) /\left(\omega_{\perp} r_{0} / \gamma\right)$. Fig. 4 shows that the parametric amplification process can be used as a sensitive probe of rotations in the torus [20], which works down to a few quanta of circulation and is complementary to the techniques discussed in [19].

All results shown above have been obtained assuming the condensate to be at $T=0$. In this case, the fluctuations which are parametrically amplified are those due to the small quantum depletion of the condensate. However the dynamics of the amplification is greatly independent of the type and origin of the initial fluctuations. What really matters is the amount of effective seed (i.e., a specific quadrature of counter-propagating Bogoliubov excitations [12]), which determines the time $t_{\text {mod }}$ needed to obtain a visible density pattern. In an experiment the imperfections in loading the condensate in the trap and thermal fluctuations can also contribute to the seed. In particular, one expects that thermal fluctuations become dominant for temperature $T \gg \hbar \Omega / k_{B}$. We have performed simulations in this regime finding that the time $t_{\text {mod }}$ needed to achieve the maximum pattern visibility, defined as in Fig. 2, scales approximately as $(1 / \Omega) \ln (\Omega / T)$. This is consistent with the fact that the growth rate and the amount of seed are expected to be proportional to $\Omega$ and $T / \Omega$, respectively [12]. For the parameters of Fig. 2 and for $T \gg \hbar \Omega / k_{B}$ a factor 2 in temperature implies a change of about $10 \mathrm{~ms}$ in the timescale of the amplification. This suggests that the visibility of the pattern as a function of $\Omega$ could provide a way to measure $T$ in a regime of temperatures where standard thermometric methods are not applicable.

In conclusion, we have shown that a periodic modulation of the confining potential of a toroidal conden- sate induces a spontaneous pattern formation through the parametric amplification of counter-rotating Bogoliubov excitations. This can be viewed as a quantum version of Faraday's instability for classical fluids in annular resonators. The occurrence of this pattern in both density and velocity distributions provides a tool for measuring fundamental properties of the condensate, such as the excitation spectrum, the amount of thermal and/or quantum fluctuations and the presence of quantized circulation and persistent currents.

[1] S. Gupta et al., Phys. Rev. Lett. 95, 143201 (2005); A.S. Arnold et al., Phys. Rev. A 73, 041606(R) (2006); S. R. Muniz et al., e-print cond-mat/0606540.

[2] W. Rooijakkers, Appl. Phys. B 78, 719 (2004); M.B. Crookston et al., J. Phys. B 38, 3289 (2005). L. Amico et al., Phys. Rev. Lett. 95, 063201 (2005).

[3] L.D. Landau and E.M. Lifshitz, Mechanics (Oxford, Pergamon, 1973); V.I. Arnold, Mathematical Methods of Classical Mechanics (Springer-Verlag, Berlin, 1989).

[4] F. Dalfovo et al., Rev. Mod. Phys. 71, 463 (1999).

[5] We checked the accuracy of this approximation in the case of a 2D annular condensate, finding that the behavior of parametric resonances and pattern formation is scarcely affected by the inclusion of centrifugal terms.

[6] C. Tozzo and F. Dalfovo, New J. Phys. 5, 54 (2003).

[7] A. Sinatra et al., J. Mod. Opt. 47, 2629 (2000); D.F. Walls and G.J. Milburn, Quantum Optics, (Springer Verlag, Berlin, 1994).

[8] We use a split-step method, combining a Fast Fourier Transform evolution and a Crank-Nicholson algorithm.

[9] L.P. Pitaevskii and A. Rosch, Phys. Rev. A 55, R853 (1997); Yu. Kagan and L.A. Maksimov, Phys. Rev. A 64, 053610 (2001); F. Chevy et al., Phys. Rev. Lett. 88, 250402 (2002).

[10] M.C. Cross and P.P. Hohenberg, Rev. Mod. Phys. 65, 851 (1993).

[11] M. Krämer et al., Phys. Rev. A 71, 061602(R) (2005).

[12] C. Tozzo et al., Phys. Rev. A 72, 023613 (2005).

[13] K. Staliunas et al., Phys. Rev. Lett. 89, 210406 (2002), and Phys. Rev. A 70, 011601(R) (2004).

[14] M. Faraday, Philos. Trans. Roy. Soc. London 299, Sec. 103 (1831).

[15] R. Keolian et al., Phys. Rev. Lett. 47, 1133 (1981).

[16] At $\Omega=\omega_{\perp}$ the second harmonics of the modulation couples to the breathing mode $2 \omega_{\perp}$. This nonlinear process causes the anomalous behavior at $\Omega \sim \omega_{\perp}$ in Fig. 2

[17] N. Katz et al., Phys. Rev. Lett. 93, 220403 (2004); C. Tozzo and F. Dalfovo, Phys. Rev. A 69, 053606 (2004).

[18] F. Zambelli and S. Stringari, Phys. Rev. Lett. 81, 1754 (1998).

[19] M. Cozzini et al., Phys. Rev. A 73, 013603 (2006).

[20] We have verified that the misalignement has a similar behavior and is still detectable also including interations during the expansion. 